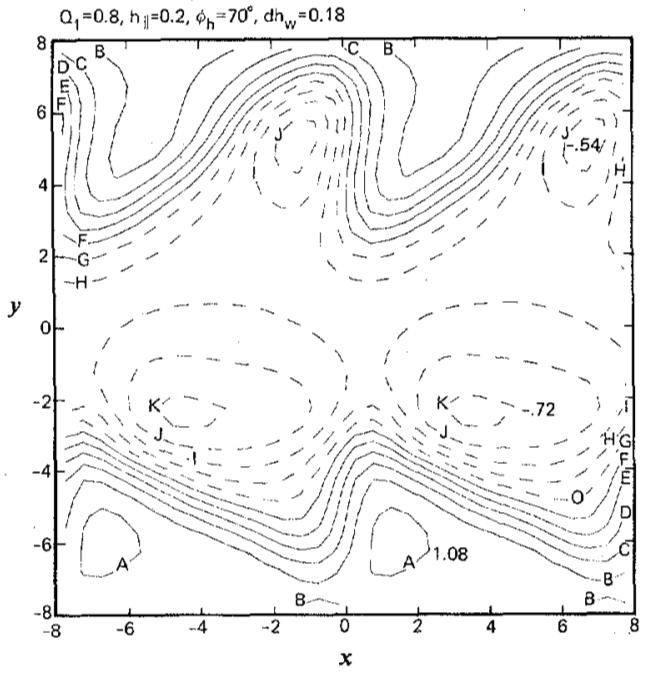

Fig. 9. Potential well distributions.

\section{DrSCUSSION}

Numerical simulations shown here can provide understanding of magnetic distributions in an implanted garnet film with complex geometry and thus supply a useful tool for device design and material parameter selection. The present results are preliminary. One problem arising from this study is the grid resolution. The charged wall width may be quite narrow at high in-plane anisotropy. The detailed structure of the wall is lost in the finite grid approximation. This problem is quite cumbersome and will be studied further.

\section{ACKNOWLEDGMENT}

The author is grateful to Dr. J. Cooley who supplied the program for fast real Fourier transform, which is an essential part of this computation, and to Mr. W. Cole for discussions about the integration of the demagnetizing field.

\section{REFERENCES}

[1] Y. S. Lin, D. B. Dove, S. Schwarzl, and C. C. Shir, IEEE Trans. Magn., vol. MAG-14, p. 494, 1978.

[2] R. Wolfe and T. J. Nelson, presented at 1978 INTERMAG Conf., paper no. 11-4.

[3] H. Jouve, J. Appl. Phys., vol. 50, no. 3, p. 2246, 1979.

[4] C. C. Shir and Y. S. Lin, J. Appl. Phys., vol. 50, no. 6, 1979.

[5] C.T.M. Chang, IEEE Trans. Magn., vol. MAG-16, no. 5, p. 952, 1980.

[6] C. C. Shir, J. Appl. Phys., vol. 49, no. 6, p. 3413, 1979.

[7] C. C. Shir and Y. S. Lin, J. Appl. Phys., vol. 50, no. 3, p. 2270 , 1979.

\title{
Determination of Magnetic Profiles in Implanted Garnets using Ferromagnetic Resonance
}

\author{
CHARLES H. WILTS, SENIOR MEMBER, IEEE, AND S. PRASAD
}

\begin{abstract}
Magnetic properties of the implanted layer in thin garnet films can be obtained from ferromagnetic resonance experiments. Approximate profiles of implantation induced anisotropy can be obtained using perpendicular resonance alone. For maximum information and accuracy, both perpendicular and parallel resonance spectra are needed, and measurements should be made on a number of samples from which varying amounts of the implanted layer have been removed by ion milling. For narrow linewidth materials it is possible to deduce profiles of $H_{k}, 4 \pi M$, and $A$ and to determine the value of $g$ in the implanted layer. Methods are presented for the accurate calculation of parallel ferromagnetic resonance (FMR) spectra with depth varying magnetic parameters. This method of analysis has been successfully applied to a yttrium iron garnet (YIG) film substituted with $\mathrm{Gd}, \mathrm{Tm}, \mathrm{Ga}$, and implanted with He ions at $140 \mathrm{keV}$ with a density of $3 \times 10^{15} \mathrm{~cm}^{-2}$.
\end{abstract}

Manuscript received March 13, 1981; revised May 13, 1981.

C. H. Wilts is with the California Institute of Technology, Pasadena, CA 91125 .

S. Prasad is with the Physics Department, Indian Institute of Technology, Bombay, India.

\section{INTRODUCTION}

ON IMPLANTATION of garnet films can be used to form guiding structures and to stabilize performance of bubble memory devices. Because of these potential uses, an understanding of the process is of practical as well as theoretical importance. Below the implantation level that destroys ferromagnetism, the principal effect of implantation appears to be a negative change in uniaxial anisotropy. Variation of other parameters is expected but is not presently understood in any detail. In the study reported here, implanted garnet films have been measured in ferromagnetic resonance (FMR) in both the perpendicular and parallel configurations. They were subsequently analyzed theoretically to confirm the validity of the differential equations used for this analysis and to explore the extent to which the depth variation of all magnetic properties can be determined by FMR. Such a study gives maximum in- 
formation for a garnet with reasonably narrow linewidth and in which implantation has been chosen to give a large variation in anisotropy without producing nonmagnetic regions. This insures a maximum number of observable surface spin-wave modes for which both mode location and FMR amplitude can be measured. In this study we were fortunate to have industrial support which provided samples with various implantation profiles. A preliminary study of similar implantations encountered difficulty in converging on magnetic profiles with satisfactory accuracy. Earlier work [1], [2] had shown the benefits of etching in reconstruction of profiles, but the uncertainty in etching depth and lack of uniform etching over the surface of a sample restricted the accuracy attainable. This problem was circumvented in part by the use of the more accurate ion-milling. Fifteen samples were provided from each implantation with varying amounts of the surface removed. The milling depth, which ranged from 0.08 to $0.9 \mu \mathrm{m}$, was determined by mechanical measurement of narrow unmilled stripes with a resolution of $0.005 \mu \mathrm{m}$ and estimated accuracy of $\pm 0.02 \mu \mathrm{m}$. Since the 15 th milling step completely removed all magnetic material, this provided an absolute measurement of thickness independent of any calculations or uncertain optical data.

The material selected was (111) oriented yttrium iron garnet (YIG) with $\mathrm{Gd}, \mathrm{Tm}, \mathrm{Ga}$ substitution giving a garnet with nearly zero value of net uniaxial anisotropy and a basic linewidth of $75 \mathrm{Oe}$ at $9.5 \mathrm{GHz}$. Implantation was with $\mathrm{He}$ ions at an energy of $140 \mathrm{keV}$ and with dose $3 \times 10^{15} \mathrm{~cm}^{-2}$. This implantation induced anisotropy changes to a depth of about $0.6 \mu \mathrm{m}$ with peak value of about 3000 Oe. In what follows, the implanted region will be called the surface layer, and the remainder of the film will be called the body or bulk region. Ferromagnetic resonance in the perpendicular configuration showed at least seven surface modes above the main body mode and two or three small lower-lying resonances presumed to be primarily body spin-wave modes. Parallel resonance showed a spectrum consisting of a main body mode with up to seven lower-lying modes that could not be separated easily into categories of body, surface, or mixed modes. Table I gives the basic magnetic parameters quoted by the supplier and their measured values of ion-milling depth.

Spectra in perpendicular and parallel FMR are shown in Fig. 1 for the unmilled sample and for a sample with the entire implanted layer removed. The discussion and analysis of these spectra will be deferred until later. The purpose in presenting them at this point is to show the wealth of experimental data available and to establish the motivation for the discussion which follows. It is clear from a casual inspection of the perpendicular FMR spectrum that there is a large change in one or more magnetic parameters within the implanted layer. From theoretical considerations the most likely candidate is a decrease in the uniaxial anisotropy $H_{k}$ of about $3000 \mathrm{Oe}$, and the structure observed in this spectrum must be primarily dependent on the variation of $H_{k}$ with depth. What is not clear is whether concomitant variations exist in the magnetization $M$, crystalline anisotropy field $H_{1}$, exchange constant $A$, damping parameter $(\alpha)$ and gyromagnetic ratio $(\gamma)$; nor is it easy to
TABLE I

Basic Magnetic Properties of Garnet and Implantation Schedule

\begin{tabular}{|c|c|c|c|}
\hline NOMINAL COMPOSITION & \multicolumn{2}{|c|}{$\left(G d .28^{\mathrm{mim}} \cdot 39^{\gamma} \cdot 33_{3}\right.$} & $\left.\mathrm{Fe}_{2}{ }^{\mathrm{Ga}} .{ }_{13} \mathrm{Fe} \cdot .87^{\prime}\right)_{3} \mathrm{O}_{12}$ \\
\hline $4 \pi M$ (gauss) & 523 & Sample No & $\begin{array}{l}\text { Miiling Depth } \\
\text { (iun) }\end{array}$ \\
\hline Nominal Thickness $(\mathrm{km})$ & .95 & 0 & 0 \\
\hline Wall Energy & .23 & $\begin{array}{l}1 \\
2 \\
3\end{array}$ & $\begin{array}{l}.075 \\
.125\end{array}$ \\
\hline Strip Width & .95 & $\begin{array}{l}3 \\
4\end{array}$ & $\begin{array}{l}.195 \\
.28\end{array}$ \\
\hline $\begin{array}{l}\text { Implantation Energy } \\
(\text { Kev })\end{array}$ & 140 & $\begin{array}{l}5 \\
6 \\
7\end{array}$ & $\begin{array}{l}.355 \\
.405 \\
.45\end{array}$ \\
\hline $\begin{array}{l}\text { Implantation Dese } \\
\left.\text { (He ions } / \mathrm{cm}^{-2}\right)\end{array}$ & $3(10)^{15}$ & $\begin{array}{l}8 \\
9 \\
10 \\
11 \\
12 \\
13 \\
14 \\
15\end{array}$ & $\begin{array}{r}.475 \\
.525 \\
.575 \\
.65 \\
.73 \\
.805 \\
.855 \\
.93\end{array}$ \\
\hline
\end{tabular}

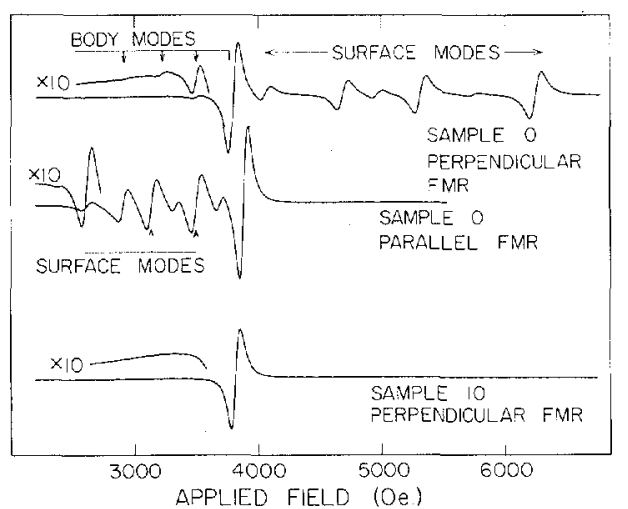

Fig. 1. Ferromagnetic resonance spectra for implanted garnet film and for one with implanted layer removed by ion-milling. See Fig. 7 for identification of parallel FMR modes.

see at first how one might separate the effects of such variations. It will be seen in what follows that a surprising amount of evidence is found in the FMR spectra if one is able to use etched or milled samples and include analysis of both perpendicular and parallel resonance configurations.

It is also a simple matter to measure resonance at other angles. For reasons both experimental and analytic, such measurements are not useful except for determination of bulk properties in the unimplanted garnet. The experimental reason is that the modes have a maximum separation for parallel and perpendicular configurations where they can be resolved most clearly and measured with maximum accuracy. From perpendicular resonance to the critical angle where the spectral lines merge to a single line, the number of modes observed and the mode spacing both decrease with no important change in mode structure. The same remark applied to measurements taken between parallel resonance and the same critical angle. The analytical reason relates to the great increase in complexity of analysis of mode structure. The major complication is the large deviation in direction of the static magnetization through the thickness of the film due to the large deviation $(\sim 3000 \mathrm{Oe})$ in anisotropy. The assumption of a constant angle is simply not sufficiently accurate at $X$-band frequencies where $(\omega / \gamma)$ is only 4000 Oe. 


\section{ANALYTICAL CONSIDERATIONS}

Given the depth dependence of all magnetic parameters, the mode locations and shapes can be calculated by solving a twopoint boundary value problem. In the case of perpendicular resonance, a single second-order equation is involved. Satisfactory methods for solving this equation are well-documented in the literature [1]-[2] and are summarized in the Appendix. A somewhat simplified form of this equation which is useful for qualitative discussion is

$$
\frac{d^{2} m}{d z^{2}}=\frac{M}{2 A}\left\{H_{a}-\frac{\omega}{\gamma}+\left(H_{k}-4 \pi M\right)-\frac{2}{3} H_{1}\right\} m .
$$

For resonance with applied field at any other angle, two coupled second-order equations are required. As discussed earlier, only parallel resonance is useful here. To date, no satisfactory method has been reported for calculation of parallel resonance with space varying magnetic properties. Two methods have been developed in the course of this work. One is an inprinciple exact numerical method; the other is a much faster approximate method using only one second-order equation. A detailed discussion of the equations and the two methods of solution are also found in the Appendix. A simplified form of the approximate equation is

$$
\frac{d^{2} m}{d z^{2}}=\frac{M}{2 A}\left\{H_{a}-\frac{\omega}{\gamma}-\frac{1}{2}\left(H_{k}-4 \pi M\right)-\frac{1}{4} H_{1}+\epsilon\right\} m
$$

where $\epsilon$ is small compared to the other terms. In these equations $H_{a}$ is the magnitude of the applied static field and $m$ is the magnitude of the radio frequency (RF) deflection of the magnetization from static equilibrium. The gyromagnetic ratio $\gamma$ is, of course, related to the $g$-factor $\left(\gamma=\mu_{0} e g / 2 m_{e}\right)$. All magnetic parameters $\left(M, H_{k}, H_{1}, A\right.$, and $\left.\gamma\right)$ may be functions of the coordinate $z$ measured normal to the surface, with some limitations discussed in the Appendix.

The important difference between (1) and (2) is the sign change in the anisotropy term. It is this feature which puts the surface modes above the uniform body mode in perpendicular resonance and below in parallel resonance. It also has another important consequence. The implantation profile often shows a peak in ion dose inside and well below the surface of the garnet film. For example, for the implantation analyzed here, this peak occurs at a depth of about $0.4 \mu \mathrm{m}$. It will be shown in later sections that the principal surface mode in perpendicular resonance probes the magnetic parameters at this point, while the first principal surface mode in parallel resonance probes the magnetic parameters at the surface. The importance of this difference should be emphasized. None of the higher order modes in perpendicular FMR give direct data regarding the surface conditions, these higher modes being affected by the entire profile.

It was recognized early in the study that it was not sufficient to match mode location, but equally necessary to match mode amplitudes. For low loss insulator ferromagnets, the mode amplitude in perpendicular resonance can be calculated using

$$
A=K_{0} \frac{\left(\int m d z\right)^{2}}{\left(\int \frac{\alpha}{\gamma M} m^{2} d z\right)} .
$$

The relative amplitudes of all modes can be obtained from this equation if the mode shapes have been obtained using (1). This equation can be generalized to resonance at any angle if the excitation method is known. It is convenient to establish resonance with the sample oriented in the microwave cavity so that the exciting magnetic field lies in the plane of the sample. In this case the mode amplitude is

$$
A=K_{0} \frac{\left(\int m_{\phi} d z\right)^{2}}{\left(\int \frac{\alpha}{2 \gamma M}\left(m_{\phi}^{2}+m_{\theta}^{2}\right) d z\right)} .
$$

In this equation, $m_{\theta}$ and $m_{\phi}$ are the polar and azimuthal components of the RF magnetization. The space-variation of $\alpha, \gamma$, and $M$, as well as the mode shape, enter into the calculation for both perpendicular and parallel FMR.

As detailed in the Appendix, the solution of (1) gives the eigenvalue $H_{a}$ as well as the mode shape. If this analysis can be used to fit the experimental data, it establishes the space dependence of both $(M / A)$ and $\left[\left(H_{k}-4 \pi M\right)-\frac{2}{3} H_{1}-\omega / \gamma\right]$. Regardless of the accuracy which might be attained, there is no way to separate the individual variation of $M$ from $A$, nor that of $\left(H_{k}-4 \pi M\right)$ from $H_{1}$ or $\omega / \gamma$. On the other hand, a similar treatment of parallel resonance can in principle give the space variation of $(M / A)$ and $\left[\left(H_{k}-4 \pi M\right)+\frac{1}{2} H_{1}+2 \omega / \gamma\right]$. Again the individual magnetic parameters cannot be separated. However the values of $(M / A)$ must be consistent, and comparison of the two bracketed quantities permits determination of the variation in two of the three parameters if one can argue convincingly that the variation of the third is negligibly small. This separation cannot be made with any assurance unless parallei resonance is used.

If the mode amplitudes from (3) and (4) can be matched to experimental data, this provides some measure of information regarding the variation of $(\alpha / \gamma M)$. The relative values of $\alpha$ can be found by comparing linewidths of body and surface modes, and calculation of resonance locations as described above gives information about the variation of $\gamma$. Combining these data permits an evaluation of the variation of $M$. Again combining this with the earlier knowledge of $(M / A)$ finally permits determination of $A$. In summary, if the linewidth is sufficiently narrow to give a number of measurable modes, and if one is successful in obtaining a fit to the experimental data with unique plausible profiles one can successfully separate the profiles or space dependence of $H_{k}, A$, and $M$, and in some cases even $H_{1}$ and $g$.

No optimum method has been found for deducing these profiles of the magnetic parameters. The sequential trial-and-error procedure used in this study is documented in the following sections. However, two comments are relevant here. The bulk properties are best obtained from uniform unimplanted samples, 
or samples from which the entire implanted area has been removed. As will be seen, this eliminates all boundary pinning conditions and permits attention to be focused on the single uniform mode without mode shape affecting amplitude or exchange shift affecting location. Here resonance should be considered at all angles so that accurate bulk values can be obtained for $\left(H_{k}-4 \pi M\right), H_{1}$, and $g$ (or $\gamma$ ) [3]. For surface layer profile studies, it is advantageous to start with perpendicular resonance. Not only are the surface modes more widely spaced, but more modes are observed experimentally, and the surface modes are not intermixed with body modes. This can be clearly seen in the spectra of Fig. 1 where the various types of modes are identified.

\section{Determination of Bulk Magnetic Parameters}

It is common practice to identify the principal resonance modes of implanted garnets as bulk uniform modes in order to obtain values for $\gamma$ (or $g$ ) and $\left(H_{k}-4 \pi M\right)$ in the main bulk of the film. If the unimplanted region is thicker than $1 \mu \mathrm{m}$, the exchange shift is less than $4 \mathrm{Oe}$ and can be safely ignored. For this series of samples, the bulk region is only $\frac{1}{4}-\mu \mathrm{m}$ thick so that the exchange shift from the uniform mode can exceed $60 \mathrm{Oe}$, and the above procedure would be inaccurate. However the ion-milling of samples 10 through 13 has removed the entire implanted layer, and only a single resonance is seen in perpendicular FMR, with no surface modes above nor bulk spin-wave modes below (see Fig. 1). This indicates that the mode observed is the uniform mode and that there is no constraint on the magnetization at either surface. In other words the spins are unpinned at both substrate interface and upper milled surface. This has a significance beyond the identification of the uniform mode, since it gives very strong evidence that this is also the proper pinning condition to be used at the top surface of the implanted and partially milled samples. If the amplitude of the FMR resonance is plotted versus milling depth, a straight line is obtained which extrapolates to zero at a milling depth which is approximately that of sample 14 . Data are available for three nearly identical wafers, each of which was separately implanted and subsequently ion-milled. Careful examination of the three number 14 samples shows no resonance for one, and for the other two, very small resonances with amplitude about 1 percent of sample 10. These data plotted in Fig. 2 establish the total thickness of the magnetic layer at $0.855 \pm 0.02 \mu \mathrm{m}$, which is 10 percent less than the nominal value of Table $\mathrm{I}$.

If only perpendicular and parallel FMR are used to measure the bulk magnetic parameters, a significant error will be made in the determination of $\gamma$ and $\left(H_{k}-4 \pi M\right)$ if $H_{1}$ is too large. For this material the magnitude of $H_{1}$ is nearly $200 \mathrm{Oe}$ and it must be included in the analysis [3]. Using the thickest bulk sample (number 10), resonance was measured as a function of the angle between the film normal and the applied field. If the film is rotated about an in-plane (1-12) axis, a symmetric variation is obtained (this fact is used in the laboratory to determine the ( $(\overline{1} 2)$ axis). If rotated about an in-plane axis perpendicular to this (or at any odd multiple of $30^{\circ}$ ), the maximum asymmetry is obtained since, $\bar{H}_{a}$ and $\bar{M}_{0}$ pass directly through a (100) axis during the rotation. Fig. 3 shows a best fit of the experimental data from which values were obtained

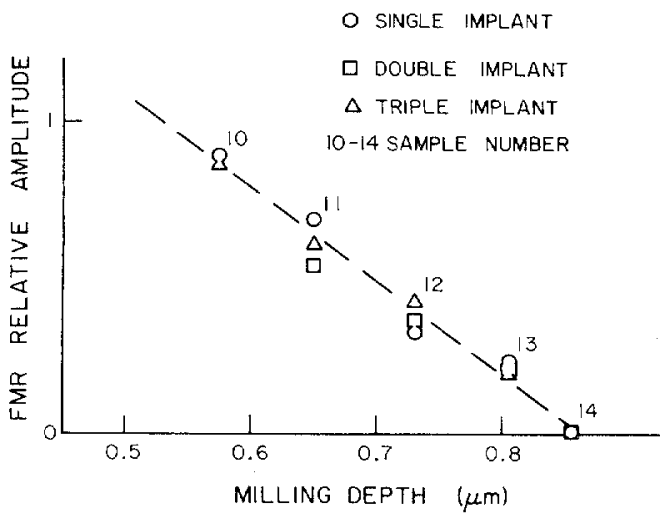

Fig. 2. Amplitude of FMR resonance versus ion-milling depth for garnet films in which entire implanted layer has been removed. Note three sets of data from three similar wafers.

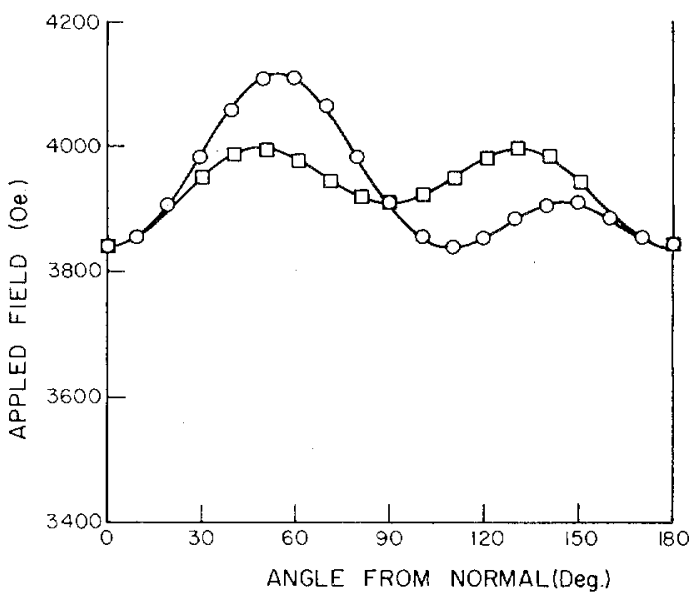

Fig. 3. Resonance location as function of angle between film normal and applied field. Implanted layer has been removed by ion-milling. $\circ$ : rotation around $(\overline{1} 10)$ axis. $\square$ : rotation around $(\overline{1} \overline{1} 2)$ axis.

for $(\omega / \gamma),\left(H_{k}-4 \pi M\right)$, and $H_{1}$ with a probable error less than \pm 10 Oe for each:

$$
\begin{aligned}
H_{k}-4 \pi M & =0 \\
H_{1} & =-165 \mathrm{Oe} \\
\omega / \gamma & =3950 \mathrm{Oe} .
\end{aligned}
$$

Since the microwave frequency is $9.49 \mathrm{GHz}$, the bulk value for $g$ is $1.717 \pm 0.010$.

Since no spin-wave modes are observed in these samples, no estimate can be made of the exchange constant $A$. However, in the spectra of the thicker samples, body spin-wave modes are found below the uniform mode location. Their amplitudes are strikingly dependent on the surface magnetic parameters, but their location is predominantly determined by the value of $A$ in the unimplanted region. From these considerations, the value of $A$ was found to be $A_{\text {bulk }}=2.47\left(10^{-7}\right) \mathrm{erg} / \mathrm{cm}$.

\section{Determination of Surface Magnetic Parameters}

Samples 0 through 9 show a varying number of surface modes in the perpendicular FMR spectra. The disappearance of all but the uniform mode in sample 10 and beyond establishes the maximum implantation depth at about $0.58 \mu \mathrm{m}$. A 
major goal of this work was to determine a specific depth variation of magnetic properties between the surface and this point that would predict the location and magnitude of all observed modes in the ten samples.

The first parameter that can be readily determined is the relative value of the damping constant $\alpha$. In all of the perpendicular FMR spectra, the main resonance had a linewidth of $75 \mathrm{Oe}$, and the surface modes showed a minimum linewidth of about 90 Oe. Since the main mode involves a magnetic excitation primarily in the bulk while the higher surface modes have an excitation exclusively in the implanted layer, this indicates that the damping parameter $\alpha$ is about 20 percent larger in the surface layer. As discussed in the next section, there is an uncertain planar variation in the implantation dose, which will give rise to a broadening of the line when it occurs within the area of a particular sample. The samples used here are small enough (3-mm square) so that the minimum linewidth can be attributed to the actual damping parameter. Presumably there should be a variation in $\alpha$ within the surface layer. Since this variation has only a minor effect on surface mode amplitudes and no effect on locations, it is a satisfactory simplification to adopt the constant value $\alpha_{\text {surf }}=1.2 \alpha_{\text {bulk }}$.

For films in which the implantation has not produced nonmagnetic layers, the major parameter determining the mode structure is the variation in anisotropy $H_{k}$. Using this as the only variable, an approximate agreement between experimental and calculated mode locations was obtained but with unacceptable discrepancies in mode amplitudes. This implied that variations in the other magnetic parameters was necessary, but because of their smaller effect poorer resolution would be expected in their profiles unless a dependence on the change in $H_{k}$ could be established. Unfortunately there is neither sufficient understanding of the implantation process nor of the ferromagnetism of strained and damaged crystals to make any confident estimate of correlative changes in the magnetic parameters. The existence of some correlation is obvious, since the Curie temperature of implanted layers is usually reduced, and continued increase in implant density ultimately destroys ferromagnetism [4] , [5]. Changes in $M$ and $A$ are likely to be greatest where the anisotropy change is greatest, and a linear relationship would be computationally convenient, but there is no firm basis for such an assumption; there may well be a threshold for which changes are minimal, with a high rate of change near the density for which magnetism disappears. Nevertheless, for initial investigation, the most straightforward assumption is that the changes in both $M$ and $A$ are proportional to the change in $H_{k}$. Should this prove to be unsatisfactory, other assumptions can be tested. Since the changes in $M$ and $A$ generally have a smaller effect on the spectrum, such refinement may not be meaningful and it may not be possible to resolve the question.

The initial synthesis of profiles of $H_{k}, M$, and $A$ was undertaken using perpendicular FMR spectra and the assumption that $H_{1}$ and $\gamma$ were unchanged in the implanted layer. The use of progressively thicker samples to build up the profiles was found essential in obtaining a good fit to experimental data. The implication here is that we were not clever enough to converge on a profile using only the unmilled spectrum, but there are two other important features of the milling experiment.
First, the use of progressively thicker samples guarantees a unique profile, if a successful synthesis can be accomplished. Second, the solution of (1) or (2) gives local values of $\left(M / A \delta^{2}\right)$ where $\delta$ is a distance parameter related to thickness locally. Without an absolute scale of distance there is no possibility of separating $A$ from $\delta$. This applies both to the bulk value and depth dependent values in the implanted layer. Only through the milling experiment can this important magnetic parameter be established with any confidence.

The synthesis of profiles of $H_{k}, M$, and $A$ was successful in the sense that each successive sample only required adjustment of the three parameters for values of $z$ beyond the thickness of the previous sample. In the final fit, the change in the ratio $(A / M)$ between surface and bulk was about 5 percent. If nearly constant values of $A$ and $M$ were used consistent with this ratio, then there were relative amplitude errors of about 70 percent between surface and bulk modes. Resolution of this discrepancy through (3) required a large decrease in $M$ in the surface layer or a similar decrease in $\gamma$.

In fitting the perpendicular FMR spectra, it had been temporarily assumed that there was no significant variation in $\gamma$. It was now necessary to use the parallel spectra to complete the synthesis and confirm the substantial decrease in $M$ with negligible change in $\gamma$. This conclusion that $g$ is not significantly affected by implantation is important from the theoretical viewpoint and deserves further discussion. The profiles chosen for $H_{k}, M$, and $A$ predicted all mode properties in perpendicular resonance and correct mode amplitudes in parallel resonance. But in the case of parallel resonance mode locations, a small but significant error (about $60 \mathrm{Oe}$ ) was found in all surface modes. It can be seen from (1) and (2) that this small shift could be attributed to an increase in $H_{1}$ of $100 \mathrm{Oe}$ (from -165 to $-65 \mathrm{Oe}$ ) or an increase in $\omega / \gamma$ of $40 \mathrm{Oe}(1.0$ percent decrease in $\gamma$ ), a shift so small that it would be pointless to look for a profile of this variation.

It is not possible to distinguish between variations in $H_{1}$ or $\gamma$ (or a suitable combination of the two) unless resonance can be accurately measured and calculated at another angle, the most favorable direction being a (100) axis. Experimentally this is particularly difficult because the variation of $H_{a}$ with angle $\beta$ near $\beta=55^{\circ}$ is so great that the required precision may not be attainable. ${ }^{1}$ Computationally, it is also difficult for reasons already cited. Without such a measurement, there remains a small uncertainty in the value of $g$ in the surface layer. However, even an unlikely change of $H_{1}$ of 100 percent would not allow more than a 2 percent change in $\gamma$. This conclusively shows that there is no significant change in $g$ in the surface layer. Any change must be at most of the order of 1 percent.

\section{Discussion of Results}

Final profiles of $H_{k}, 4 \pi M$, and $A$ are shown in Fig. 4, and other magnetic parameters are listed in Table II. Profiles could be suggested for $\alpha$ and for $H_{1}$, but the variations are so slight that no experimental basis exists for these profiles nor would it affect the calculated mode structure in a significant way.

\footnotetext{
${ }^{1}$ Note this problem arises because of the very large value of $H_{k}$ in the implanted layer. It does not exist in the bulk material, as can be seen in Fig. 3.
} 

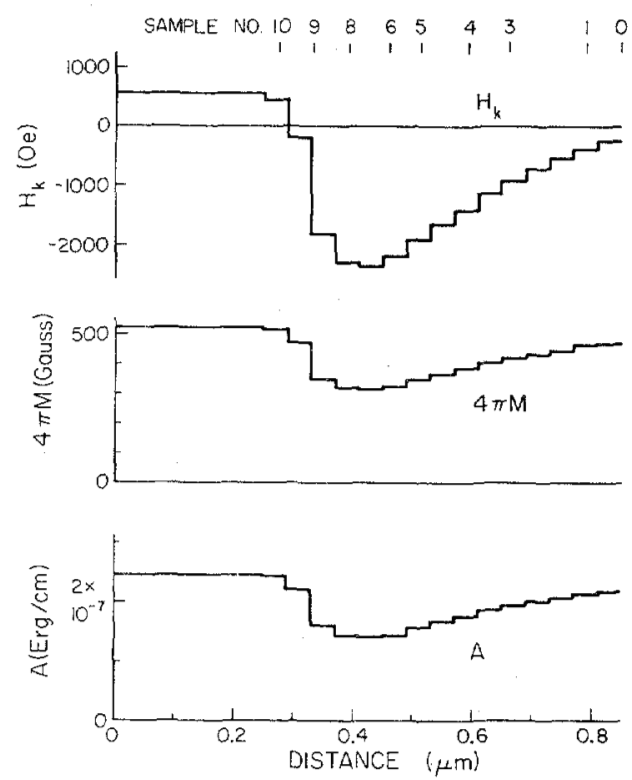

Fig. 4. Profiles of $H_{k}, 4 \pi M$, and $A$ as function of depth in implanted layer.

TABLE II

Magnetic Parameters of Implanted Garnet Deduced from FMR SPECTRA

\begin{tabular}{|c|c|c|}
\hline & Bulk Data & Surface Data \\
\hline $4 \pi M$ & 523 gauss & (see Fig. 4) \\
\hline y & $7.506(10)^{7}(\sec 0 e)^{-1}$ & $1.506(10)^{7}$ * \\
\hline A & $2.47(10)^{-7}\left(\mathrm{erg} \mathrm{cm}^{-1}\right)$ & 2.47 to $7.41(10)^{-7}$ (see Fig. 4 ) \\
\hline $\mathrm{H}_{\mathrm{T}}$ & $-1650 e$ & -60 \\
\hline$H_{k}$ & 543 oe & 543 to $-2330 *$ (see Fig. 4 ) \\
\hline$\alpha$ & .05 & .06 \\
\hline \multicolumn{3}{|c|}{$\begin{array}{l}\text { Equivalent values: } Y=1.491(10)^{7}, H_{1}=-165,\left(H_{k}\right)_{\min }=-2360 \\
\text { See text for discussion. }\end{array}$} \\
\hline \multicolumn{3}{|c|}{$\begin{array}{l}\text { Profiles of } 4 \pi M, A \text { and } H_{k} \text { are given in Fig. } 4 \text {. The range of values is } \\
\text { given in the table above. }\end{array}$} \\
\hline
\end{tabular}

Comparison of experimental and calculated mode structure is given in Figs. 5 and 6 . In these figures the calculated mode locations are shown by solid lines as continuous functions of ion-milling depth. Experimental data are shown by circles at the value of thickness where the experimental and calculated spectra were matched. Since the experimental values of ionmilling depth are not precise, the match point was allowed to deviate slightly from the experimental depth where necessary. Comparison with Table I shows a random deviation up to $\pm 0.02 \mu \mathrm{m}$, consistent with the estimated precision of the ionmilling depths. Where circles are absent, the experimental mode is either so small that it could not be observed or it was so close to a large mode that its location could not be determined even though its existence was evident. Mode amplitudes, both experimental and calculated, are shown at the plotted location of each experimental mode. The calculated mode amplitude is also given at those points where a mode is predicted but not observed experimentally. In those cases where a missing mode is well separated from the other modes, the calculated amplitude is always very small (see, for example, mode 6 for sample 0 , mode 10 for sample 1 , and mode 2 for sample 4 in Fig. 5). Where a small mode lies within a linewidth of a large mode, it cannot be measured experimentally even when as large as 5 or 10 percent (for example, see mode 7 , sample 3 in Fig. 5 and mode 4, sample 1 in Fig. 6).

There is an interesting difference in mode structure between perpendicular and parallel FMR. For the perpendicular configuration, all surface modes are located above the uniform mode $H_{\text {un }}$ and involve negligible excitation in the bulk. The body spin-wave modes are located below $H_{\text {un }}$ and involve a significant excitation in the surface layer. The main mode is either a surface mode just above $H_{\text {un }}$ or a body mode just below $H_{\text {un }}{ }^{2}$ or frequently it is not a single mode but a closely

${ }^{2}$ One cannot logically define a surface mode as one which is "confined" to the surface layer, since with properly chosen ion-milling one can get any degree of penetration in to the bulk layer. The only logical definition for perpendicular resonance is that the mode shape in the bulk be hyperbolic for surface modes, sinusoidal for bulk modes, and linear for the uniform mode. This distinction is impossible in parallel resonance so that designation of surface or body mode is now qualitative. 


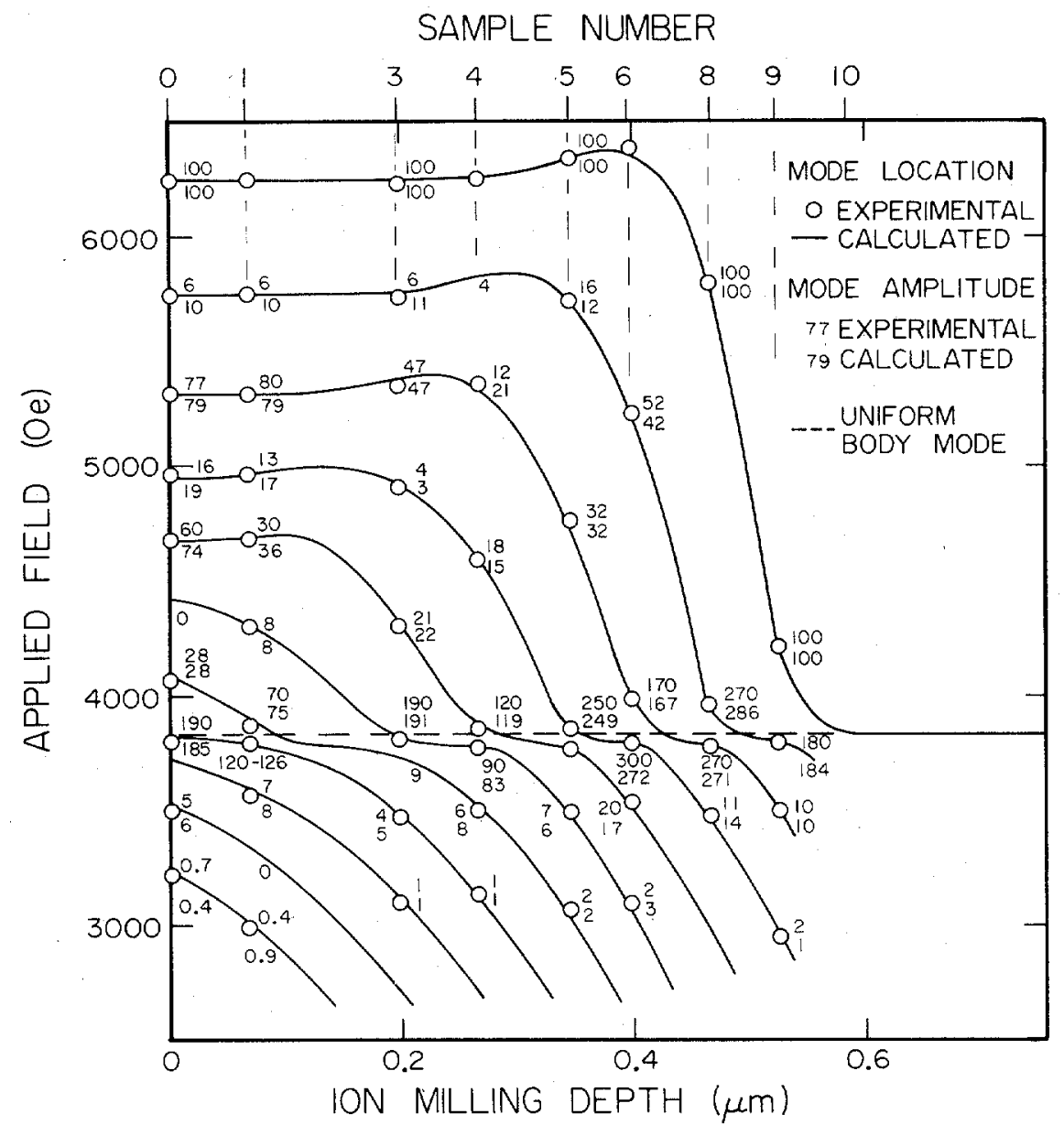

Fig. 5. Comparison of location and amplitude of experimental and calculated modes in perpendicular resonance. This includes as-implanted film and eight thinner samples with varying depths of ion-milling.

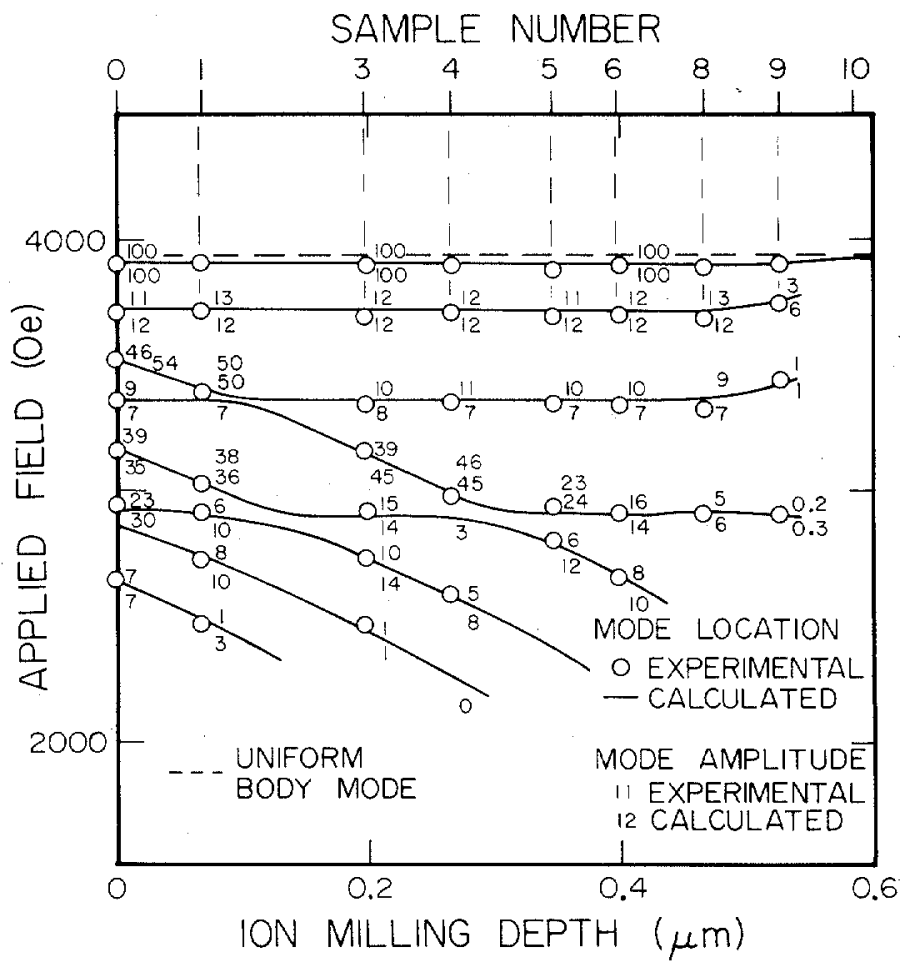

Fig. 6. Comparison of location and amplitude of experimental and calculated modes in parallel resonance. This includes as-implanted film and eight thinner samples with varying depths of ion-milling. 
spaced pair. Examples of all three can be easily found in Fig. 5 where the uniform mode location is 3830 Oe. The number of surface modes is seven for the unmilled garnet, and this number decreases monotonically to zero when the ion-milling distance exceeds the implantation depth. The body spin-wave modes are generally very small except for the ion-milling distance of about $0.4 \mu \mathrm{m}$ where one of them becomes as large as 20 percent (both experimentally and theoretically).

In parallel FMR the structure is quite different. The highest mode is a body mode, lying very close to the parallel uniform mode. The exchange shift here is only about 25 Oe. The lower lying modes are initially predominantly surface or body modes with negligible excitation in the other region, but beyond the fifth mode, they are of a mixed nature. In Fig. 6, the third mode is strictly a surface mode for the unmilled sample and it moves downward as milling progresses, becoming the fourth mode and finally the fifth mode as it crosses below one or another of the body modes. The fifth mode is also a surface mode and shows similar behavior.

To show the character of these modes clearly, the calculated mode shapes of the entire spectrum are shown for the unmilled garnet in Fig. 7. The highest ten modes are shown in perpendicular FMR, and the highest eight modes in parallel FMR, since these encompass the modes seen experimentally. Actually, mode 6 in perpendicular FMR is not seen since its amplitude is zero, and mode 7 in parallel FMR can be seen but not measured. Even though its theoretical amplitude is 5 percent, it lies too close to mode 6 for an experimental measurement.

Mode 1 in perpendicular resonance and mode 3 in parallel resonance demonstrate an important feature mentioned earlier. In the first, the mode is localized at the region where the anisotropy has the largest negative value, and in the second it is localized at the surface. This means that the first mode measures the uniform perpendicular mode location for the maximum anisotropy variation, less the exchange shift due to the local curvature of $m$. The second measures the uniform parallel mode for the surface anisotropy, again less the appropriate exchange shift. These two modes immediately give approximate values for the anisotropy at these two points, but the parallel resonance calculation is an essential part of the analysis.

The determination of magnetic parameters described above may sound like a complicated process, and indeed it is, but the development of suitable programs for a high-speed computing facility makes it a reasonable procedure. Unfortunately, the accuracy of the result is directly dependent on the accuracy of spectra determination and the consistency of the samples. With linewidth of 75 to $90 \mathrm{Oe}$, it is an easy matter to determine the location of major surface resonances with a precision of 1 or 2 Oe if a nuclear magnetic resonance (NMR) probe is used for the field determination. However, the temperature coefficient of the exchange and the implantation anisotropy produces a variation in surface mode locations as large as 10 Oe per degree $\mathrm{C}$ so that temperature stabilization of the cavity is necessary for high accuracy. Even worse, the implantation. process is far from uniform over a wafer, and variations of more than 100 Oe in mode location were observed in supposedly identical samples. Variations of this magnitude render

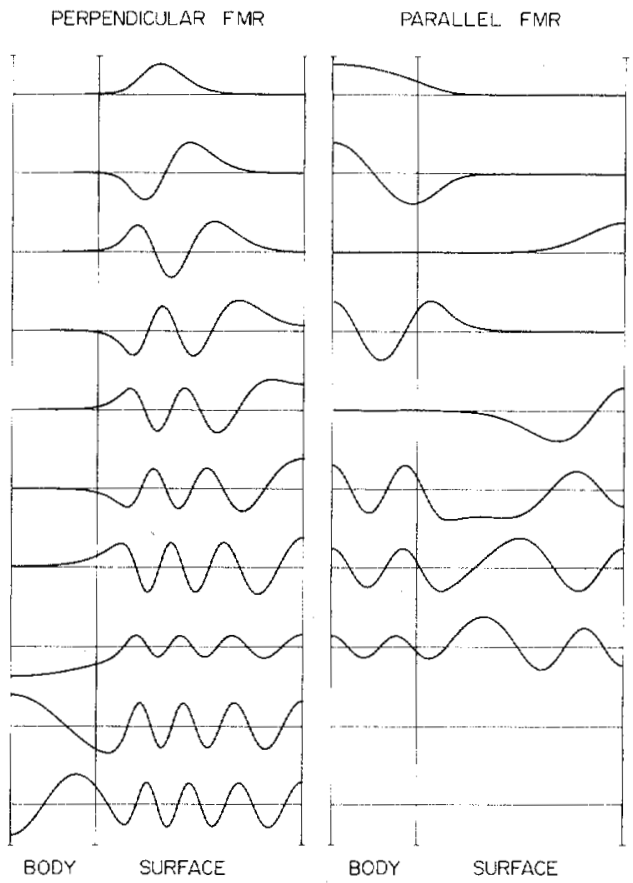

Fig. 7. Calculated mode shapes for seven surface modes and first three body modes in perpendicular FMR and first eight modes in parallel FMR-unmilled garnet.

correlation in the profile between various milled samples impossible. However, with careful selection and elimination of faulty samples, those used here are believed to have uniformity corresponding to about 30 to 40 Oe in mode location. In addition, the resonant cavity used in this work did not have temperature stabilization. The implication is that any deviaations between calculated and experimental spectra which are less than 30 Oe are of no significance.

In spite of the above limitations, the profile of $\left(H_{k}-4 \pi M\right)$ can be obtained to an accuracy of a few percent. The effect of $A, M$, and $\alpha$ variations on the spectra is somewhat smaller, and similar accuracy in these profiles is impossible. However, with the simplifying assumption that the profiles are similar (i.e., $\Delta A$ and $\Delta M$ are roughly proportional to $\Delta H_{k}$ ), then the average or overall value of $A$ and $M$ in the implanted region can be obtained with an accuracy of about 10 percent.

\section{SUMMARY}

If the FMR linewidth is sufficiently narrow, an approximate anisotropy profile can be obtained for implanted garnet films using only the perpendicular FMR spectrum. If it is desired to obtain an accurate profile for the anisotropy as well as implantation layer values for the other magnetic parameters such as $M, A$, and $g$, then it is necessary to measure FMR spectra of milled samples as well as to include parallel resonance spectra in the analysis. For some profiles, the principal surface mode in perpendicular and parallel resonance gives direct evidence of the maximum anisotropy deviation and the surface anisotropy, respectively, without resorting to a detailed analysis of the entire spectra. The method developed herein has been used to characterize the implanted layer of a Gd, Tm, Ga substituted YIG, where implantation with He ions at an energy of 140 $\mathrm{keV}$ and at a dose of $3 \times 10^{15} \mathrm{~cm}^{-2}$ gave rise to a change in 
$H_{k}$ of $2000 \mathrm{Oe}$, and a decrease in $M$ and $A$ in excess of 40 percent.

Two methods have been developed for calculating the parallel resonance mode structure. One is exact in the sense that a numerical calculation will produce solutions with error below any preselected value or tolerance. The other is an approximate method which is computationally much faster. For typical garnets the approximate method gives mode locations with error up to 4 or $5 \mathrm{Oe}$, a value which is generally of no significance experimentally.

\section{APPENDIX \\ Calculation of FMr Spectra}

Calculation of resonance in a thin layer is a two-point boundary value problem which in its simplest form involves one second-order equation for perpendicular and two coupled second-order equations for parallel configuration. The eigenvalue is usually the applied field, corresponding to the experimental situation where the frequency is held constant and the applied field is varied. The perpendicular case usually responds well to conventional "shooting" techniques where one starts at one boundary and integrates the solution until it diverges seriously or the second boundary is reached. The eigenvalue estimate is adjusted on successive iterations to converge on the value that will satisfy the final boundary condition. The simplest convergence scheme involving halving of intervals requires 10 to 15 iterations to get accuracy of 1 to $0.01 \mathrm{Oe}$. The principal difficulty in the integration is describing the $z$ dependence of the magnetic parameters since this dependence is an empirical rather than analytical variation. Most analysts use either connected straight line segments or a step function. Although the former may seem preferable at first glance, there are many distinct advantages in using a step function, and if the number of steps is sufficiently large, there is no deterioration in accuracy. For this work, the surface layer was divided into 15 regions. Test cases with 10 to 20 layers showed no significant deterioration in accuracy for the modes in question when the number exceeded 12.

The basic equation for perpendicular resonance is

$$
\frac{d^{2} m}{d z^{2}}=\frac{M}{2 A}\left\{H_{a}-\frac{\omega}{\gamma}-4 \pi M+H_{k}-\frac{2}{3} H_{1}\right\} m .
$$

This equation is essentially correct if the losses are small $(\alpha<$ 0.1 ) and only $H_{k}$ varies with $z$. When $M$ and $A$ are functions of $z$, additional terms must be added which involve $\left(d^{2} M\right) /$ $\left(d z^{2}\right)$ and $d A / d z$. The effect of these terms is small, but one is never sure of the resulting accuracy if they are omitted. Inclusion of these terms is a significant complication in the computer program and computation time. When a layered structure or step function is used, the boundary condition between layers takes care of the variation in $M$ and $A$ in a much simpler way. This boundary condition is

$$
\begin{gathered}
\frac{m_{1}}{M_{1}}=\frac{m_{2}}{M_{2}} \\
\frac{A_{1}}{M_{1}} \frac{d m_{1}}{d z}=\frac{A_{2}}{M_{2}} \frac{d m_{2}}{d z} .
\end{gathered}
$$

Furthermore, with a stepped structure, the solution in each layer is sinusoidal or hyperbolic, and it is not necessary to use a step-by-step integration procedure. ${ }^{3}$ The result is a much faster computer program, coupled with a better treatment of variation of all magnetic parameters.

For parallel resonance the relevant equations are

$$
\begin{aligned}
& \frac{d^{2} m_{\theta}}{d z^{2}}=\frac{M}{2 A}\left\{\left(H_{a}+4 \pi M-H_{k}-\frac{1}{2} H_{1}\right) m_{\theta}-\left(\frac{\omega}{\gamma}\right) m_{\phi}\right\} \\
& \frac{d^{2} m_{\phi}}{d z^{2}}=\frac{M}{2 A}\left\{H_{a} m_{\phi}-\left(\frac{\omega}{\gamma}\right) m_{\theta}\right\} .
\end{aligned}
$$

Again these equations are correct if only $H_{k}$ varies with $z$, and additional terms are required if $A$ and $M$ are also functions of $z$. Attempts to solve these equations by a similar shooting technique have not been satisfactory. Solution of the equations involves two eigenvalues-for example, the applied field and $\left(m_{\phi} / m_{\theta}\right)$ the ellipticity of $\bar{m}$ at one boundary. To properly interpret the divergences of the integration and choose new estimates of the eigenvalues, it was necessary to rewrite the equations in two new variables

$$
\begin{aligned}
y & =\left(m_{\theta}+m_{\phi}\right) / 2 \\
x & =\left(m_{\theta}-m_{\phi}\right) / 2 \\
\frac{d^{2} y}{d z^{2}} & =\frac{M}{2 A}\left\{\left(H_{a}-\frac{\omega}{\gamma}+H_{e q}\right) y+H_{e q} x\right\} \\
\frac{d^{2} x}{d z^{2}} & =\frac{M}{2 A}\left\{\left(H_{a}+\frac{\omega}{\gamma}+H_{e q}\right) x+H_{e q} y\right\}
\end{aligned}
$$

where

$$
H_{e q}=\left(2 \pi M-\frac{H_{k}}{2}-\frac{H_{1}}{4}\right) \text {. }
$$

This method is satisfactory when $y$ is much larger than $x$ (as is the case for garnet materials) because it gives a coherent pattern to the observed divergence of the integration. Whether the first divergence occurs in $x$ or $y$ permits a correct logical procedure for the next estimate of the two eigenvalues. However, the required accuracy of the ellipticity eigenvalue invariably exceeded the precision of the digital computer (even in double precision) and special treatment was necessary to prevent $x$-divergences (ellipticity uncertainties) from vitiating a solution.

Our attention was drawn to a relaxation technique advocated by Keller [6] to solve very general boundary value problems. A particularly effective program called PASVA (variable step) has been developed by two of his colleagues, Pereyra and Lentini [7]. When this method is used for a system of equations with a few finite discontinuities, a multiplexing scheme

\footnotetext{
${ }^{3}$ In principle, the same scheme could be used with connected line segments provided $M$ and $A$ were independent of $z$. In each layer the solution would be a sum of the two Airy functions. However, the difficulty of calculating the Airy functions and their derivatives makes this procedure slower than step-by-step integration if many intervals are involved. Further, it is not applicable when $M$ and $A$ are depth dependent.
} 
TABLE III

Comparison of Exact and Approximate Solutions in Parallel Resonance

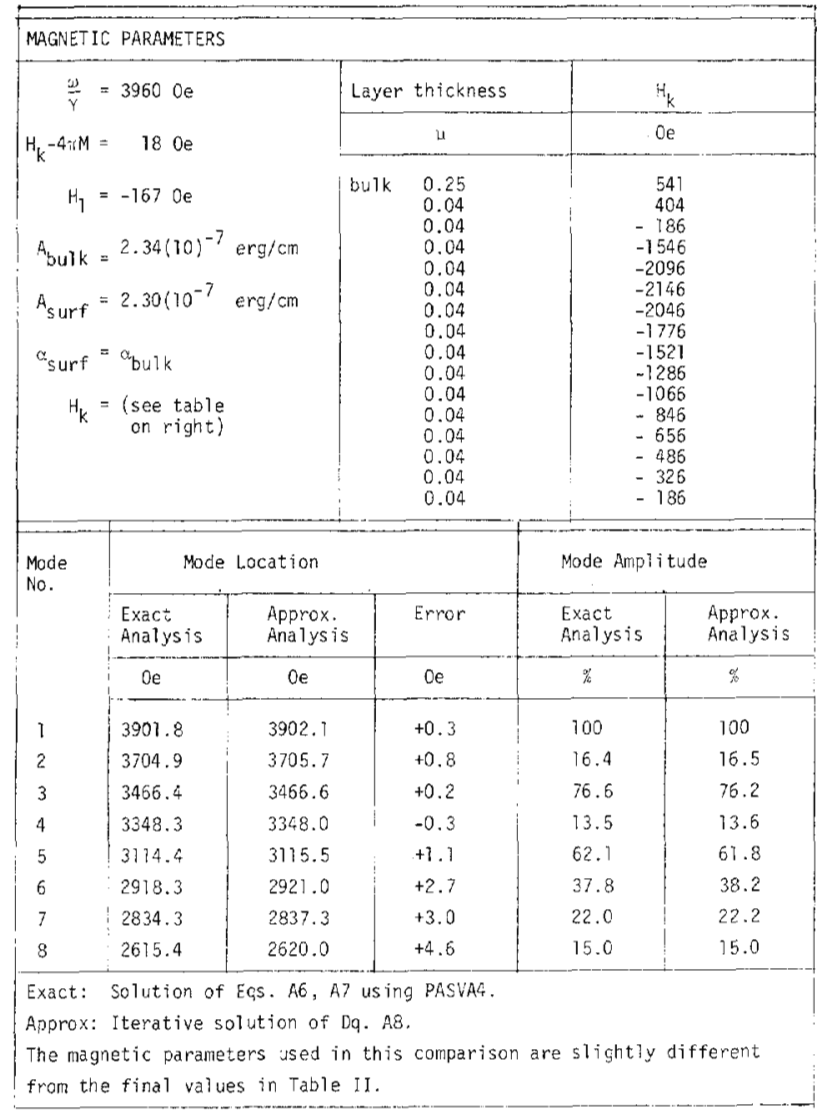

can be used to eliminate the discontinuities, i.e., to remove them to the boundaries. The multiplexing required for a structure with many steps proved completely intractable since excessive memory was required and computation time became unreasonably long. To solve this problem in a satisfactory way, it was necessary for Pereyra and Lentini to develop a new version of PASVA to allow discontinuous functions. This version, called PASVA4 [8] has been highly successful in solving either (A3) and (A4) or (A6) and (A7). The solution is, in principle, exact in the sense that the program will produce a solution with error less than any prescribed value (unless limited by the number of significant digits in the computer). For the present study any mode can be found with an accuracy of 0.01 Oe in 3 to $10 \mathrm{~s}$ of central processing unit (CPU) time with an IBM $370-3032$ system. Needless to say, this represents a very long computation, but it is orders of magnitude shorter than the earlier procedure.

If one notes that $x<<y$ in (A6) and (A7), it follows that the principal driving term in (A7) which determines $x$ is $\left(H_{e q} y\right)$ and $\left(d^{2} x / d z^{2}\right)$ is very small. An approximate solution to the two equations can be obtained by setting the second derivative $d^{2} x / d z^{2}$ to zero, solving (A7) for $x$ and substituting this in (A6):

$$
\frac{d^{2} y}{d z^{2}}=\frac{M}{2 A}\left\{H_{a}-\frac{\omega}{\gamma}+H_{e q}-\frac{H_{e q}^{2}}{\left(H_{a}+(\omega / \gamma)+H_{e q}\right)}\right\} y .
$$

This equation can now be solved by the methods used for perpendicular FMR.

The principal concern here is of course the validity of the approximation. Fortunately, one can use PASVA4 at any time to check the accuracy, but can retain the speed and convenience of this approximation in searching for profiles that give a fit to experimental spectra. Test cases have been used throughout the range of this study and have in all cases given accuracy better than $1 \mathrm{Oe}$ in the low order modes and better than $5 \mathrm{Oe}$ in the highest order modes. An example is given in Table III. In view of the experimental uncertainties, errors of $5 \mathrm{Oe}$ are considered insignificant. Needless to say, when the two methods are used in perpendicular FMR, they give identical solu. tions since no approximation is involved in either case.

\section{ACKNOWLEDGMENT}

The garnet films and ion-milling of these films was provided by the San Jose, CA, Laboratory of the IBM Corporation, whose interest in and support of this work is gratefully acknowledged.

\section{REFERENCES}

[1] B. Hoekstra, R. P. Van Stapele, and J. M. Robertson, "Spin wave resonance spectra in inhomogeneous bubble garnet films," $J$. Appl. Phys., vol. 48, p. 382, 1977.

[2] C. H. Wilts, J. Zebrowski, and K. Komenou, "Ferromagnetic resonance study of the anisotropy profile in implanted bubble garnets," J. Appl. Phys., vol. 50, p. 5878, 1979.

[3] A. Gangulee and R. J. Kobliska, "Magnetocrystalline anisotropy in epitaxially grown garnet thin films," J. Appl. Phys., vol. 51, p. $3333,1980$.

[4] K. Komenou, J. Zebrowski, and C. H. Wilts, "Ferromagnetic resonance study of the anisotropy field and nonmagnetic regions in implanted layers of bubble garnet films," J. Appl. Phys., vol. 50, p. $5442,1979$.

[5] C. W. Searle and I. Marteense, "Magnetic transition temperature of ion-implanted layers on bubble garnet films," J. Appl. Phys., vol. 51, p. $5919,1980$.

[6] A. B, Keller, "Accurate difference methods for nonlinear twopoint boundary value problems," SIAM, J. Num. Anal, vol. 11, p. $305,1974$.

[7] M. Lentini and V. Pereyra, "An adaptive finite difference solver for nonlinear two-point boundary problems with mild boundary layers," SIAM J. Num. Anal., vol. 14, p. 91, 1977.

[8] - "PASVA4-An ordinary boundary solver for problems with discontinuous interfaces and parameters," to be published. 